\title{
EFEITOS DO ETHEPHON SOBRE A PRODUÇÃO DA UVA 'NIAGARA ROSADA' (Vitis labrusca L.), PRODUZIDA NA ENTRESSAFRA NA REGIÃO DE JALES-SP ${ }^{1}$
}

\author{
ANTONIO AUGUSTO FRACARO ${ }^{2}$, FERNANDO MENDES PEREIRA³ ${ }^{3}$ JAIR COSTA NACHTIGAL ${ }^{4}$, \\ JOSÉ CARLOS BARBOSA ${ }^{5}$
}

\begin{abstract}
RESUMO- A produção de 'Niagara Rosada' na entressafra, embora sendo uma excelente opção para os viticultores de Jales-SP, é problemática devido à dificuldade de emissão e desenvolvimento das brotações após a poda, realizada nos meses de ocorrência de temperaturas inferiores a $10^{\circ} \mathrm{C}$, o que tem causado pequenas produções e desestímulo entre os viticultores. Visando a solucionar este problema, foram conduzidos seis experimentos nos anos de 2001 e 2002, cujo objetivo foi verificar o efeito do ethephon aplicado antes da poda sobre o número de cachos, a produção e a qualidade da uva. Foram testadas as doses $0 ; 3 ; 6$ e $9 \mathrm{~L} \cdot \mathrm{ha}^{-1}$ de ethephon aplicado via foliar. Concluiu-se que o uso de ethephon proporcionou aumento do número de cachos e da produtividade de 'Niagara Rosada', especialmente quando da ocorrência de condições climáticas desfavoráveis e com satisfatório grau de enfolhamento. A utilização de ethephon na dose de 9L.ha ${ }^{-1}$ proporcionou aumento da produtividade da uva 'Niagara Rosada' na entressafra. Termos de indexação: Vitis sp, fitorreguladores, brotação, ethephon.
\end{abstract}

\section{EFFECT OF ETHEPHON ON PRODUCTION OF 'NIAGARA ROSADA' (Vitis labrusca L.), PRODUCED OFF SEASON IN THE REGION OF JALES-SP}

\begin{abstract}
The production of 'Niagara Rosada' in the off season is an excellent option for the region of Jales, São Paulo State, Brazil, due the difficulty of emission and development of the sprout after the pruning, carried through in the months of occurrence of lower temperatures $\left(<10^{\circ} \mathrm{C}\right)$, that has caused small productions among the grape growers. So to solve this problem, six experiments in the years of 2001 and 2002 had been conducted with the objective to verify the effect of ethephon applied before the pruning, on the number of clusters and production. Four levels $\left(0 ; 3 ; 6\right.$ and $\left.9 \mathrm{~L} . \mathrm{ha}^{-1}\right)$ of ethephon applied by foliar via had been tested. It was concluded that the use of ethephon provided the increasing of the number of clusters and the productivity of 'Niagara Rosada', especially with the occurrence of favorable climatic conditions and with satisfactory degree of foliage. The use of ethephon in the dose of 9L.ha- ${ }^{-1}$ provided to increase the productivity in 'Niagara Rosada' in the period between harvests.
\end{abstract}

Index Terms - Vitis sp, growth regulator, sprout, ethephon.

\section{INTRODUÇÃO}

Atualmente, a videira é cultivada em diversas regiões do mundo, sendo a Europa responsável por $51 \%$ da produção mundial de uvas, seguida pela Ásia (21\%), América (19\%) e África (5\%) (Corrêa et al., 2000).

A viticultura brasileira abrange uma área de 63.816ha, sendo o Estado do Rio Grande do Sul a maior região vitícola do país com 30.373ha de uvas, as quais $80 \%$ são de uvas americanas (Vitis labrusca L.). Nos Estados de Pernambuco e Bahia, a uva ocupa 8.000ha. No Estado do Paraná ocupa 3.953,1ha, sendo 499ha de 'Niagara Rosada', em Santa Catarina 1.706,9ha, Minas Gerais 755,3ha, sendo 20ha de 'Niagara Rosada'. O Estado de São Paulo utiliza 9.082ha na produção de uvas, sendo 5.270,2ha de 'Niagara Rosada', sendo que a região Noroeste deste Estado possui 1.212ha, dos quais 108,5ha são da mesma cultivar. (Protas et al., 2002).

A 'Niagara Rosada', que é produzida na região de Jales-SP, no período de entressafra, durante os meses de setembro-outubro, alcança preços acima de $\mathrm{R} \$ 1,80 \mathrm{~kg}^{-1}$.

Esta produção encontra problemas, principalmente relacionados à dificuldade de emissão e desenvolvimento das brotações após as podas de produção realizadas quando da ocorrência de temperaturas inferiores a $10^{\circ} \mathrm{C}$, normalmente entre maio e julho. Tal fato provoca redução na produção e, conseqüentemente, desestímulo dos viticultores por esta cultivar.

$\mathrm{Na}$ tentativa de solucionar esses problemas, podem-se utilizar reguladores de crescimento, em especial o ethephon. Porém, o modo de ação é dependente do local da síntese ou tecido aplicado, do tempo de síntese ou da aplicação, do nível de ação do composto, bem como a da sua interação e a inter-relação funcional de diferentes hormônios e reguladores de crescimento (Korban, 1998).
Lavee (1987), Lavee et al. (1984), Seyijewicz et al. (1984), Mannini (1982), citados por Bautista et al. (1987), relataram que o ethephon é uma substância que libera lentamente etileno.

A síntese de etileno é influenciada por fatores como temperatura, teor carbônico, oxigênio e está correlacionada à presença de outros hormônios e reguladores vegetais (Schiaparelli et al., 1995).

A biossíntese inicia com o aminioácido metionina, que reage com ATP para formar um composto conhecido por S-adenosilmetionina (SAM), sendo quebrado em dois compostos diferentes, um dos quais é o chamado ACC (ácido 1-aminociclopropano-1-carboxílico), onde enzimas no tonoplasto convertem o ACC em etileno (Raven et al., 2001).

A utilização de ethephon em videira 'Rubi' realizada por Fracaro (2000), na região Noroeste do Estado de São Paulo, resultou em significativo aumento na intensidade de brotação e na produtividade quando as plantas foram pulverizadas com $5 \mathrm{~L} \cdot \mathrm{ha}^{-1}$, cerca de 20 dias antes da poda de produção.

Embasado nesse resultado, iniciaram-se pesquisas com ethephon na cultivar 'Niagara Rosada', visando a dizimar esse problema, de grande importância para a expansão da cultura, não somente na região, mas em quaisquer regiões tropicais e subtropicais produtoras dessa espécie.

O objetivo deste trabalho foi de verificar o efeito de diferentes concentrações de ethephon, aplicado via foliar antes da poda de produção, no número de cachos, na produção e na qualidade da uva 'Niagara Rosada', na região Noroeste do Estado de São Paulo.

\section{MATERIALEMÉTODOS}

A área experimental foi implantada em cinco propriedades na região de Jales, Noroeste do Estado de São Paulo, latitude $20^{\circ} 16^{\prime}$ S,

\footnotetext{
${ }^{1}$ (Trabalho 102/2003). Recebido: 04/09/2003. Aceito para publicação: 02/04/2004. Parte da tese de doutorado do primeiro autor à FCAV/UNESP. Projeto financiado pela FAPESP.

2 Doutorando em Agronomia, Departamento de Produção Vegetal, Universidade Estadual Paulista-UNESP, Faculdade de Ciências Agrárias e Veterinárias- FCAV, Câmpus de Jaboticabal, Rodovia de Acesso Prof. Paulo Donato Castellane, s/n, CEP14884-900, Jaboticabal-SP. Bolsista CNPq. Fone 17 3621 6884. Email:fracaro@melfinet.com.br

${ }^{3}$ Dr., Prof. Titular Aposentado, Departamento de Produção Vegetal, FCAV/UNESP, Câmpus de Jaboticabal, Jaboticabal-SP.

${ }^{4}$ Dr., Pesquisador Embrapa Uva e Vinho, Estação Experimental de Jales-SP.

${ }^{5}$ Dr., Prof. Titular, Departamento de Ciências Exatas, FCAV/UNESP, Câmpus de Jaboticabal, Jaboticabal-SP.
} 
longitude $50^{\circ} 33^{\prime} \mathrm{O}$ e altitude média de $483 \mathrm{~m}$, em vinhedos comerciais da cultivar Niagara Rosada, espaçados de 2,5 x 2,0m no sistema latada, tendo como porta-enxertos IAC 572-Jales. Foram realizados três experimentos no ano de 2001 e repetidos no ano de 2002.

O clima da região é classificado como CWa. A precipitação pluvial média anual é de $1.280 \mathrm{~mm}$ distribuídos principalmente durante os meses de agosto a abril. A estação seca ocorre entre os meses de maio e setembro. Apresenta evapotranspiração média anual de $2.205 \mathrm{~mm}$ e evapotranspiração de $234,10 \mathrm{~mm}$. A temperatura média anual é de $22,3^{\circ} \mathrm{C}$, com média das mínimas de $19,9^{\circ} \mathrm{C}$ e média das máximas de $29,0^{\circ} \mathrm{C}$. A umidade relativa média é de $69 \%$, com máxima em março (76\%) e mínima em setembro (61\%) (Boliani, 1994).

O solo predominante da região está classificado como Podzólico Vermelho-Amarelo. O relevo na região é suave-ondulado e ondulado (Terra et al., 1998).

O delineamento experimental utilizado foi em blocos ao acaso, com quatro doses de ethephon e cinco repetições, de uma planta por parcela. Os tratamentos utilizados foram: 1)Testemunha (sem aplicação de ethephon); 2) 3L.ha- ${ }^{-1}$;)6L.ha- ${ }^{-1}$, e 4)9L.ha ${ }^{-1}$ do produto comercial contendo $240 \mathrm{~g} . \mathrm{L}^{-1}$ de ethephon.

No ano de 2001, os tratamentos foram aplicados em plantas com $30 \%$ de enfolhamento, via foliar, com pulverizador costal até o ponto de escorrimento (1.000 litros por hectare), nos dias 22-05, 30-05 e 06-06, podando-se as plantas nos dias 11-06, 18-06 e 25-06, respectivamente, para os experimentos 1; 2 e 3 . No ano de 2002, os tratamentos foram aplicados nas plantas com $80 \%$ de enfolhamento, com pulverizador até o ponto de escorrimento, nos dias 25-05, 06-06 e 17-06, podando-se as plantas nos dias 12-06, 22-06 e 03-07, respectivamente, para os experimentos $1 ; 2 \mathrm{e} 3$.

A avaliação do experimento foi realizada através da contagem do número total de cachos por parcela, e a produção foi obtida em quilograma por parcela e transformado em quilograma por hectare. Os dados foram analisados através de regressão polinomial, pelo programa SAS. Todos os tratos culturais utilizados foram os convencionais adotados para a cultura na região.

\section{RESULTADOSE DISCUSSÃO}

\section{Efeito sobre o número de cachos}

Na Figura 01, são apresentados os resultados dos experimentos realizados no ano de 2001, sobre plantas pouco enfolhadas (30\%).

Durante o desenvolvimento destes experimentos, a baixa intensidade de frio ocorrida não interferiu negativamente no desenvolvimento da brotação e, conseqüentemente, no número de cachos por planta.

A análise de todos os dados disponíveis sobre nossas



FIGURA 1 - Efeito da aplicação de ethephon antes da poda de produção sobre o Número Médio de Cachos por Planta da videira 'Niagara Rosada', cultivada na região Noroeste do Estado de São Paulo. Jales-SP, 2001. experimentações, relativa ao tema, indica que temperatura abaixo de $10^{\circ} \mathrm{C}$, no momento da brotação, afeta negativamente a emissão de brotos e seu desenvolvimento da uva 'Niagara Rosada', conseqüentemente, o número de cachos e a produção.

Verifica-se que o ethephon não propiciou diferenças estatísticas significativas em relação ao número de cachos, embora haja uma clara tendência de aumento nesta variável com o aumento na dose de ethephon aplicado. A tendência de formação de um número maior de cachos por planta no tratamento 9L.ha ${ }^{-1}$, provavelmente, foi consequiência de uma brotação mais efetiva ocorrida.

Fracaro (2000) verificou a mesma tendência observada neste trabalho, ou seja, o aumento do número de cachos com o aumento da dose de ethephon aplicada, com conseqüente aumento da produção.

Em todos os experimentos, o tratamento-testemunha apresentou um número razoável de cachos, sendo um pouco menor no experimento 3 , devido, provavelmente, à ocorrência de menor número de gemas brotadas. Entretanto, todos os tratamentos com ethephon apresentaram resultados superiores ao tratamento-testemunha.

Pelas regressões ajustadas, verifica-se que, em média, houve aumento de 3,31 cachos/planta, para cada litro de ethephon aplicado.

Verifica-se, na Figura 2, que ocorreu o aumento gradativo do número de cachos por planta com o aumento das doses de ethephon, nos três experimentos realizados no ano de 2002.

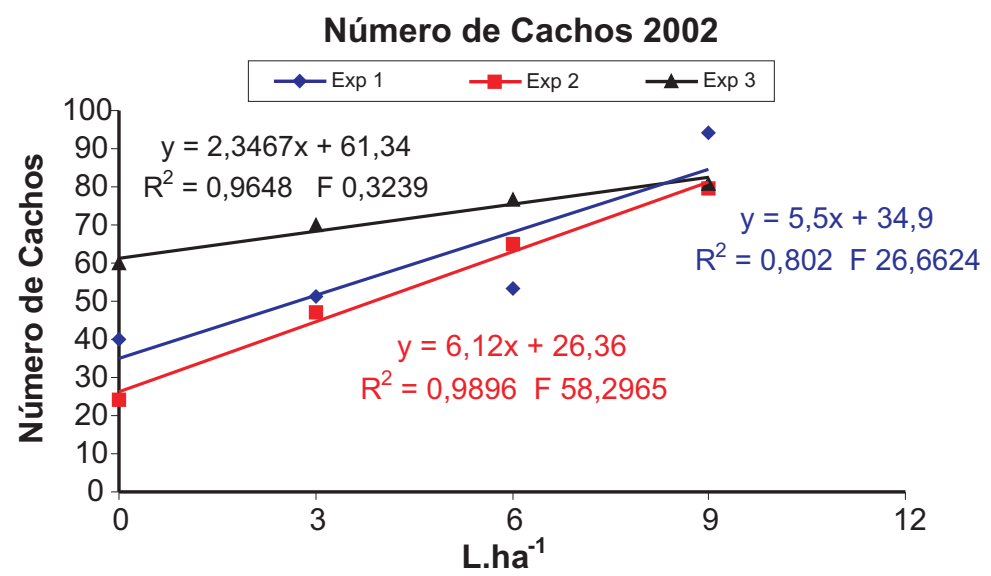

FIGURA 2 - Efeito da aplicação de ethephon antes da poda de produção sobre o Número Médio de Cachos por Planta da videira 'Niagara Rosada', cultivada na região Noroeste do Estado de São Paulo. Jales-SP, 2002.

A análise conjunta dos dados relativos ao número de cachos, nos três experimentos de 2002, evidenciou que o tratamento 9L.ha- ${ }^{-1}$ foi superior aos demais. Os tratamentos-testemunha sempre se mostraram inferiores, indicando a necessidade da aplicação do ethephon antes da poda para uma satisfatória produção de cachos durante o desenvolvimento das videiras, em período de alta incidência de frio.

No ano de 2002, a aplicação de ethephon mostrou-se muito mais efetiva quando da ocorrência de frio durante a brotação e desenvolvimento das videiras (exp. 1 e 2). Entretanto, em períodos quentes (normais), sua ação mostrou-se menos efetiva, porém muito satisfatória, o que pode ser observado no experimento 3 .

Fracaro (2000) observou forte tendência de aumento no número de cachos por planta com o aumento da dose de ethephon aplicada em uva 'Rubi', na região de Jales-SP, Noroeste do Estado de São Paulo.

Pelas regressões ajustadas, verifica-se que, em média, houve um aumento de 4,66 cachos/planta, para cada litro de ethephon aplicado.

\section{Efeitos sobre a Produção}

Observam-se, na Figura 03, os resultados da produção em quilos por hectare dos três experimentos conduzidos no ano de 2001. Os experimentos 1 e 2 não apresentaram diferenças estatísticas significativas entre os tratamentos, apesar de apresentarem forte tendência de aumento 
da produção com a utilização de ethephon. O experimento 3 apresentou diferença estatística significativa entre os tratamentos, sendo a dose de $6 \mathrm{~L} . \mathrm{ha}^{-1}$ a que apresentou o melhor resultado, equivalente a $21.140 \mathrm{~kg} . \mathrm{ha}^{-1}$.

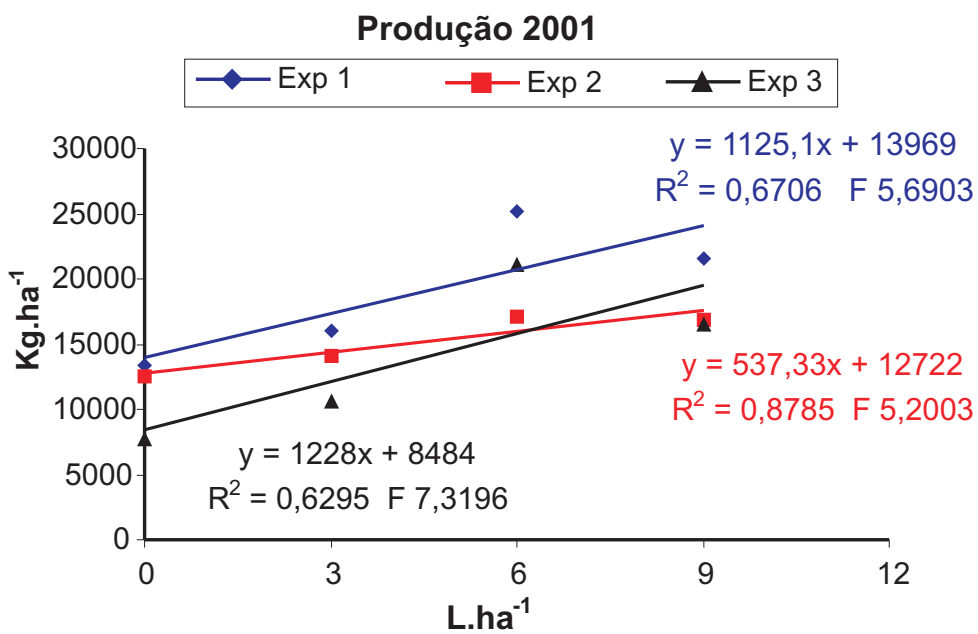

FIGURA 3 - Efeito da aplicação de ethephon antes da poda de produção sobre a Produção da videira 'Niagara Rosada', cultivada na região Noroeste do Estado de São Paulo. Jales-SP, 2001

Apesar de os dois primeiros experimentos não apresentarem diferenças estatísticas significativas entre os tratamentos, os resultados dos três experimentos seguiram a mesma tendência (Figura 3 ), ou seja, os tratamentos com as maiores doses (6 e 9L.ha-1) proporcionaram as melhores produções. Portanto, observou-se que o uso do ethephon contribuiu para aumentar a produção, mesmo em plantas com apenas $30 \%$ de enfolhamento e com ausência de temperaturas baixas, prejudiciais ao desenvolvimento da videira. Pelas regressões ajustadas, verifica-se que, em média, houve aumento de $963,48 \mathrm{~kg} \cdot \mathrm{ha}^{-1}$, para cada litro de ethephon aplicado.

Fracaro \& Boliani (2001) observaram que houve aumento da produção, quando da aplicação de ethephon em maiores doses, sobre a uva 'Rubi' em Jales-SP e obtiveram acréscimo na ordem de 31,38\% sobre a produção.

Os aumentos na produção ocorridos entre a testemunha e o tratamento $6 \mathrm{~L} \cdot \mathrm{ha}^{-1}$ foram de $11.740 \mathrm{~kg} \cdot \mathrm{ha}^{-1}, 4.580 \mathrm{~kg} . \mathrm{ha}^{-1}$ e $13.400 \mathrm{~kg} \cdot \mathrm{ha}^{-1}$, respectivamente, nos experimentos $1 ; 2$ e 3 . Esses aumentos correspondem a um incremento de $\mathrm{R} \$ 23.480,00, \mathrm{R} \$ 9.160,00$ e $\mathrm{R} \$ 26.800,00$ por hectare, considerando-se o preço médio de $\mathrm{R} \$ 2,00$ por quilo.

Verifica-se, pela Figura 4, que, nos três experimentos conduzidos no ano de 2002, ocorreu o aumento gradativo da produção com o aumento da dose de ethephon.

Observa-se uma forte tendência de aumentar a produtividade com o aumento da dose. Em média, para cada litro de ethephon aplicado,

\section{Produção 2002}

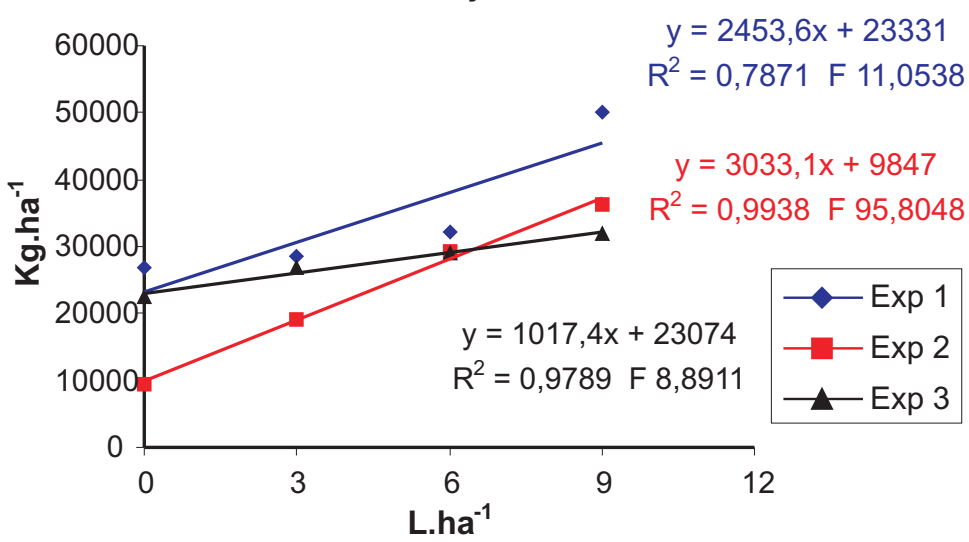

FIGURA 4 - Efeito da aplicação de ethephon antes da poda de produção sobre a Produção da videira 'Niagara Rosada', cultivada na região Noroeste do Estado de São Paulo. Jales-SP, 2002. houve um aumento de $2.168 \mathrm{~kg} \cdot \mathrm{ha}^{-1}$.

No experimento 1, os resultados evidenciaram uma produção crescente a partir do tratamento-testemunha. O tratamento com 9L.ha-1, com produtividade de $50.080 \mathrm{~kg} \cdot \mathrm{ha}^{-1}$, foi estatisticamente superior à testemunha $\left(26.780 \mathrm{~kg} \cdot \mathrm{ha}^{-1}\right)$. O uso de ethephon nas doses de $3 \mathrm{~L} \cdot \mathrm{ha}^{-1} \mathrm{e}$ 6L.ha ${ }^{-1}$ não diferiram estatisticamente do tratamento-testemunha, apesar do aumento na produção verificada. Os tratamentos com $6 \mathrm{~L} . \mathrm{ha}^{-1} \mathrm{e}$ 9L.ha ${ }^{1}$ de ethephon não diferiram estatisticamente.

No experimento 2, o mais atingindo pelas baixas temperaturas, o tratamento-testemunha apresentou a mais baixa produção, com $9.390,6 \mathrm{~kg} \cdot \mathrm{ha}^{-1}$, em relação aos demais experimentos, conseqüência da má brotação e do pouco desenvolvimento dos ramos. O tratamento com $3 \mathrm{~L} \cdot \mathrm{ha}^{-1}$ diferiu significativamente dos tratamentos com 6L.ha ${ }^{-1}$ e $9 \mathrm{~L} \cdot \mathrm{ha}^{-1}$, sendo que estes últimos não apresentaram diferença estatística entre si. $\mathrm{O}$ tratamento com 9L.ha- ${ }^{-1}$ apresentou diferença altamente significativa em relação à testemunha, com produção de $36.279,40 \mathrm{~kg} \cdot \mathrm{ha}^{-1}$.

A deficiente brotação devido à ocorrência de baixas temperaturas proporcionava baixa rentabilidade à cultura da uva 'Niagara Rosada' na região.

Fazendo-se uma análise conjunta dos experimentos conduzidos nos anos de 2001 e 2002, foi possível verificar que a utilização do ethephon, na dose de 9L.ha-1 ${ }^{-1}$ é uma prática que pode auxiliar o viticultor a reduzir perdas com aumento e regularidade na produção de 'Niagara Rosada'.

É importante ressaltar que o uso de ethephon deve estar associado ao uso adequado de outras práticas no pomar, principalmente de manejo, adubação e controle fitossanitário, para que a produção de 'Niagara Rosada' seja otimizada em regiões tropicais e subtropicais do Brasil.

Como a aplicação de ethephon é realizada em torno de 20 dias antes da poda de produção e não se pode saber com certeza sobre a ocorrência ou não de frio, a utilização deverá ser necessária para a garantia de boa emissão de brotos e a formação de um número satisfatório de cachos mesmo não ocorrendo temperaturas prejudiciais à brotação e ao desenvolvimento dos ramos e, conseqüentemente, à produtividade.

Em todos os experimentos conduzidos, não foram verificados sintomas de danos às plantas devido às aplicações com ethephon, mesmo nas doses mais elevadas.

\section{CONCLUSÕES}

Nas condições em que foram desenvolvidos os experimentos, foi possível concluir que:

1. O uso de ethephon proporcionou aumento do número de cachos e da produtividade da uva 'Niagara Rosada', especialmente quando da ocorrência de temperaturas inferiores a $10^{\circ} \mathrm{C}$ e com satisfatório grau de enfolhamento.

2. A utilização de ethephon, na dose de 9L.ha-1 ${ }^{-1}$, proporcionou o mais significativo aumento na produtividade da uva 'Niagara Rosada' na entressafra.

\section{REFERÊNCIAS BIBLIOGRÁFICAS}

BAUTISTA, A.D.; VARGAS, G.; COLMENARES, J.C. Influencia del etefon sobre la brotacion y fertilidad de tres cultivares de vid. Agronomia Tropical, Maracaju, v. 41, n. 5-6, p. 225-35, 1987.

BOLIANI, A.C. Avaliação fenológica de videiras Vitis vinifera L. cv. Itália e cv. Rubi, na região noroeste de São Paulo. 1994. 188f. Tese (Doutorado em Produção Vegetal) - Faculdade de Ciências Agrárias e Veterinárias, Universidade Estadual Paulista, Jaboticabal, 1994.

CORRÊA, L. de S.; BOLIANI, A.C. O cultivo de uvas de mesa no Brasil e no mundo e sua importância econômica In: SIMPÓSIO BRASILEIRO SOBRE UVAS DE MESA, 2000, Ilha Solteira. Anais... p. 1-34.

FRACARO, A.A. Efeito de doses crescentes de ethephon em videira 'Rubi' (Vitis vinifera L.), cultivada na região Noroeste do Estado de 
São Paulo. 2000. 88f. Dissertação (Mestrado em Sistema de Produção) - Faculdade de Engenharia de Ilha Solteira, Universidade Estadual Paulista, Ilha Solteira, 2000.

FRACARO, A.A.; BOLIANI, A.C. Efeito do ethephon em videira 'Rubi' (Vitis vinifera L.), cultivada na região Noroeste do Estado de São Paulo. Revista Brasileira de Fruticultura, Cruz das Almas, v. 23, n. 3, p. 510-512, 2001.

KORBAN, S.S. Influence of growth regulators on fruit plant growth and development. In: CONGRESSO BRASILEIRO DE FRUTICULTURA, 15., 1998, Poços de Caldas. Anais... Lavras:SBF, 1998. p. 56-81.
PROTAS, J.F.S.; CAMARGO, U.A.; MELO, L.M.R. A viticultura brasileira: realidade e perspectivas In: SIMPÓSIO MINEIRO DE VITICULTURA EENOLOGIA, 1., 2002, Caldas. Anais... Caldas:EPAMIG 2002. p. 1732.

RAVEN, P.H.; EVERT, R.F.; EICHHORN, S.E. Biologia vegetal. 6.ed. Rio de Janeiro: Ed. Guanabara Koogan. 2001. 872p.

TERRA, M.M.; PIRES, E.J.P.; NOGUEIRA, N.A.M. Tecnologia para produção de uva 'Itália' na região Noroeste do Estado de São Paulo. Campinas:CATI, 1998. 81p. (Documento técnico, 97). 